\title{
THE RING OF H I IN LEO: \\ PROBING THE MASS DISTRIBUTION IN A GALAXY GROUP
}

\author{
Stephen E. Schneider, Edwin E. Salpeter, Yervant Terzian \\ Department of Astronomy, Cornell University
}

The intergalactic neutral hydrogen in the M96 group (Schneider et al. 1983) provides an unusual probe of the detailed mass distribution in a group of galaxies. Previous observations (Schneider 1985) tentatively suggested the existence of a large ring structure to the gas. Sensitive new observations made at Arecibo confirm that the intergalactic gas forms a 200 kpc diameter* eccentric ring around the two central galaxies in the group, the giant elliptical galaxy NGC 3379 (M105) and the lenticular NGC 3384.

The pattern of measured radial velocities around the ring matches that of a noncircular Keplerian orbit with a uniquely defined focus. Without any assumptions about the focus of the orbit, the Keplerian model predicts a position and velocity for the center of mass closely coinciding with the two central galaxies. A mass distribution leading to significant deviations from a $1 / r^{2}$ force law over the length scale of the ring (and likewise Milgrom's theory of gravitation) can neither account for a closed eccentric orbit nor the ability of the Keplerian model to predict the galaxies' position and velocity. The enclosed gravitational mass for the orbit is $\sim 6 \times 10^{11} \mathrm{M}_{\odot}$, leading to a modest mass-to-light ratio of $\sim 25$ for the total mass associated with the two galaxies out to $\sim 100 \mathrm{kpc}$. This value for $M / L$ exceeds internal estimates, based on the two galaxies' stellar velocity dispersions, by only a factor of $\sim 2$ (Tonry and Davis 1981).

There are some deviations from the Keplerian model at the ring's $70 \mathrm{kpc}$ pericenter that might be explained by a halo distribution of matter about the central galaxies; however, these deviations may also result from M96's tidal influence, and the success of the Keplerian model implies that a massive halo, if present, must not extend much farther than the pericenter distance from the central galaxies. Hot massive neutrinos or any other form of dark matter spread evenly throughout the group are unlikely sources for any significant contribution to the group's mass; and a mass distribution not extending much beyond the luminous disks of the galaxies appears to be consistent with the data.

\section{REFERENCES}

Schneider, S. E. 1985, Ap. J. (Letters) 288, L33.

Schneider, S. E., Helou, G., Salpeter, E. E., and Terzian, Y. 1983 Ap. J. (Letters) 273, L1. Tonry, J. L., and Davis, M. 1981 Ap. J. 246, 666.

* Values quoted are for an assumed distance of $10 \mathrm{Mpc}$. Distance estimates for the M96 group range from 8 to $18 \mathrm{Mpc}$. 